\title{
Research on Harmonic Analysis in Power Systems Based on Neural Network
}

\author{
Rui-peng Yang \\ Department of Electrical Engineering,North China Electric Power University,Baoding,China \\ 524347954@qq.com
}

Keywords: Harmonic analysis; Electric power system; Neural network; Algorithm; Computer simulation

\begin{abstract}
A new harmonic analysis method for electric power systems based on triangle basis functions neural network was presented, the convergence theorem of the algorithm was proposed, and a window function and interpolation algorithm were employed to correct the frequency of fundamental waves. This approach does not require synchronized sampling and integer period truncation, and can obtain directly the frequencies, amplitudes and phases of fundamental waves and harmonics. The result of computer simulations has shown that the algorithm is an ideal analysis method with high precision, small amount of calculation and speedy convergence.
\end{abstract}

\section{Introduction}

With the development of power electronics technology, power system harmonic pollution is worsening, which poses a potential threat to the power system security, stability, economic operation and greatly affects the surrounding electrical environment. Therefore, it is significant to monitor the power system harmonics real-timely, exactly control the status of the power system harmonic, prevent harmonic harm and maintain safe operation of power system[1].

The power system harmonic measurement usually using fast Fourier transform (FFT) to realize. However, the power system frequency is not necessarily rated frequency. It is unable to guarantee the sampling frequency is an integer multiple of the actual operating frequency. Thus, there exists a fence effect and leakage phenomenon, which make the calculated signal parameters, namely the frequency, amplitude and phase, incorrect, especially the phase error is large, which can not meet the harmonic measurement requirements. This paper presents a new method adapted to the power system harmonic analysis based on a triangular basis functions neural network algorithm. The neural network algorithm convergence theorem is proposed and proved. The simulation indicates that the method of calculating harmonic measurements is of high precision, small amount of calculation, fast convergence, so the algorithm has a higher electric system harmonic measurement application value [2,3].

\section{The extraction of power system fundamental frequency}

A periodic signal having a respective harmonic can be expressed as:

$$
y(t)=\sum_{n=0}^{N} A_{n} \sin \left(2 \pi f_{n} t+\varphi_{n}\right)
$$

Where $f_{n}$ represents the frequency of $n$ harmonic; $A_{n}$ and $\varphi_{n}$ respectively represent the amplitude and phase of $n$ harmonic; $N$ represents highest harmonic frequency. 
The signal is sampled at a sampling frequency $f_{s}$, when the sampling frequency $f_{s}$ is not an integer multiple of the fundamental frequency $f_{0}$, the fundamental frequency can be expressed as:

$$
f_{0}=\left(K_{0}+\Delta K_{0}\right) f_{s} / M
$$

Where $K_{0}$ is integer; $\Delta K_{0}$ is decimal; $M$ is sampling points number.

According to the relevant literature, the correction equation of fundamental frequency combined with short form windowed interpolation algorithm can be obtained.

$$
\Delta K_{0}=\left\{\begin{array}{l}
\frac{Y\left(K_{0}+1\right)}{Y\left(K_{0}\right)+Y\left(K_{0}+1\right)}, Y\left(K_{0}+1\right) \geq Y\left(K_{0}-1\right) \\
\frac{Y\left(K_{0}-1\right)}{Y\left(K_{0}\right)+Y\left(K_{0}-1\right)}, Y\left(K_{0}+1\right)<Y\left(K_{0}-1\right)
\end{array}\right.
$$

And the correction equation of fundamental frequency combined with Hanning window interpolation algorithm can also be obtained.

$$
\Delta K_{0}=\left\{\begin{array}{l}
\frac{2 Y_{w}\left(K_{0}+1\right)-Y_{w}\left(K_{0}\right)}{Y_{w}\left(K_{0}\right)+Y_{w}\left(K_{0}+1\right)}, Y_{w}\left(K_{0}+1\right) \geq Y_{w}\left(K_{0}-1\right) \\
\frac{Y_{w}\left(K_{0}\right)-2 Y_{w}\left(K_{0}-1\right)}{Y_{w}\left(K_{0}\right)+Y_{w}\left(K_{0}-1\right)}, Y_{w}\left(K_{0}+1\right)<Y_{w}\left(K_{0}-1\right)
\end{array}\right.
$$

Where $Y_{w}\left(K_{0}\right)$ represents the windowed discrete Fourier transform.

Thus, the Hanning window correction fundamental frequency can be obtained from Eq. (2).

\section{Power system harmonic analysis based on triangle basis neural network}

In the power system, Eq. (1) can be further expressed as:

$$
y(m)=A_{0}+\sum_{j=1}^{N}\left[A_{j} \sin \varphi_{j} \cos \left(j w_{0} m T_{s}\right)+A_{j} \cos \varphi_{j} \sin \left(j w_{0} m T_{s}\right)\right]
$$

Where $w_{0}$ is the fundamental angular frequency of the power system, $w_{0}=2 \pi f_{0} ; j$ is the number of harmonic; $A_{0}$ is the DC component.

Eq. (5) can be further expressed as:

$$
y(m)=w_{0}+\sum_{j=1}^{N} w_{j} \cos \left(j w_{0} m T_{s}\right)+\sum_{j=N+1}^{2 N} w_{j} \sin \left[(j-N) w_{0} m T_{s}\right]
$$

Where $m$ represents the $m$ th sampling point, $m=0,1, \cdots, M-1 ; T_{s}$ represents the sampling period.

According to Eq. (6), the BP neural network model based on triangular basis function can be established. Wherein the input layer neurons is 1 , the weight value of the hidden layer neurons is 
$w_{j}$, and the excitation function is $c_{j}$, namely:

$$
\begin{aligned}
& w_{j}=\cos \left(j w_{0} m T_{s}\right), \quad j=0,1,2 \cdots, N \\
& c_{j}=\sin \left[(j-N) w_{0} m T_{s}\right], \quad j=N+1, N+2, \cdots, 2 N
\end{aligned}
$$

The weight matrix is defined as:

$W=\left[w_{0}, w_{1}, \cdots, w_{2 N}\right]^{T}$

The excitation matrix is defined as:

$C=\left(c_{0}, c_{1}, \cdots, c_{2 N}\right)$

Thus, the output of neural network is obtained:

$y_{d}(m)=\sum_{j=0}^{2 N} w_{j} c_{j}=W^{T} C$

The error function:

$e(m)=y(m)-y_{d}(m), \quad m=0,1,2, \cdots, M-1$

The performance index:

$$
J=\frac{1}{2} \sum_{m=0}^{M} e^{2}(m)
$$

The weight adjustment:

$$
W(m+1)=W(m)+\eta_{e}(m) C(m)
$$

Where, $\eta$ represents the learning rate, and $0<\eta<1$.

In order to ensure the convergence of neural networks, we propose a convergence theorem:

Set $\eta$ as the learning rate, when $0<\eta<2 /(N+1)$, the neural network is of convergence, where $2 N+1$ is the number of hidden layer neurons.

According to the above neural network algorithm, through neural network training, the weigh value of neural network $W$ can be obtained, and the amplitude and the phase of each harmonic can be obtained by using the weigh value $W$ and the following equation.

Fundamental amplitude: $A_{1}=\sqrt{w_{1}^{2}+w_{N+1}^{2}}$.

Fundamental phase: $\varphi_{1}=\arctan \left(w_{1} / w_{N+1}\right)$.

$n$th harmonic amplitude: $A_{n}=\sqrt{w_{n}^{2}+w_{N+n}^{2}}$.

$n$th harmonic phase: $\varphi_{n}=\arctan \left(w_{n} / w_{N+n}\right)$.

\section{Simulation example}

In order to verify the neural network algorithm proposed in this paper, take simulation harmonic 
analysis to the following signal:

$$
y(k)=\sum_{n=1}^{11} A_{n} \sin \left(2 \pi f_{n} k T_{s}+\varphi_{n}\right)+0.01 A_{1} \cdot \operatorname{rand}(k)
$$

Where the fundamental frequency is $50.2 \mathrm{~Hz}$. The harmonic frequency of each other is the integer multiples of the fundamental frequency. The sampling frequency is $1500 \mathrm{~Hz}$ and the sampling points is 1024 points. The fundamental, the harmonic amplitudes (a unit-value) and phase are shown in Table. 1.

Table. 1 Components of the simulated harmonic signal

\begin{tabular}{ccccccccccccc}
\hline Simulation & & \multicolumn{10}{c}{ Harmonic number } \\
signal & Fundamental & 2 & 3 & 4 & 5 & 6 & 7 & 8 & 9 & 10 & 11 \\
\hline Amplitude & 1 & 0.02 & 0.1 & 0.01 & 0.05 & 0.05 & 0.04 & 0 & 0.03 & 0 & 0.02 \\
Phase & 0 & 10 & 20 & 30 & 40 & 50 & 60 & - & 80 & - & 100 \\
\hline
\end{tabular}

In order to simulate the actual digital signal, the white noise signal whose amplitude is the $1 \%$ of the fundamental amplitude is added in the simulation signal. At first, obtain the fundamental frequency, through using the windowed interpolation algorithm described earlier, and regard this fundamental frequency as the one used in neural network algorithm. Put the sampling value (30 points) of the previous fundamental period into the neural network to train. After six iterations, the neural network is of convergence, namely, the amplitude and phase of the fundamental harmonic and other harmonic can be obtained with one-time. Table. 2 is the result of proposed neural network algorithm with rectangle-windowed interpolation algorithm. Table. 3 is the result of proposed neural network algorithm with Hanning-windowed interpolation algorithm. For comparison, we use adaptive artificial neural network algorithm for harmonic analysis of this signal, the analysis results are shown in Table. 4.

Table. 2 The result of proposed neural network algorithm with rectangle-windowed interpolation algorithm

\begin{tabular}{ccccccc}
\hline $\begin{array}{c}\text { Harmonic } \\
\text { number }\end{array}$ & Frequency & $\begin{array}{c}\text { Relative } \\
\text { error }\end{array}$ & Amplitude & $\begin{array}{c}\text { Relative } \\
\text { error }\end{array}$ & Phase & $\begin{array}{c}\text { Relative } \\
\text { error }\end{array}$ \\
\hline 1 & 50.1996035 & -0.0007894 & 1.00000333 & 0.01033 & 0.00118116 & 0.0006562 \\
2 & 100.3992070 & -0.0007894 & 0.01999150 & 0.042500 & 10.01175094 & 0.1175094 \\
3 & 150.5988105 & -0.0007894 & 0.09999463 & -0.005370 & 20.00670294 & 0.0335174 \\
4 & 200.7944140 & -0.0007894 & 0.00999567 & -0.043300 & 30.03567769 & 0.1189256 \\
5 & 250.9980175 & -0.0007894 & 0.04999686 & -0.006280 & 40.01318266 & 0.0329567 \\
6 & 301.1976210 & -0.0007894 & 0.00499773 & -0.045400 & 50.07577618 & -0.1515524 \\
7 & 351.3942245 & -0.0007894 & 0.03999794 & -0.005150 & 60.01820710 & 0.0303452 \\
9 & 451.7964315 & -0.0007894 & 0.02999893 & -0.003567 & 80.02506330 & 0.0313166 \\
11 & 552.1956385 & -0.0007894 & 0.02000029 & 0.001450 & 100.03820892 & 0.0382089 \\
\hline
\end{tabular}


Table. 3 The result of proposed neural network algorithm with Hanning-windowed interpolation algorithm

\begin{tabular}{ccccccc}
\hline $\begin{array}{c}\text { Harmonic } \\
\text { number }\end{array}$ & Frequency & $\begin{array}{c}\text { Helative } \\
\text { error }\end{array}$ & Amplitude & $\begin{array}{c}\text { Relative } \\
\text { error }\end{array}$ & Phase & $\begin{array}{c}\text { Relarmonic phase } \\
\text { error }\end{array}$ \\
\hline 1 & 50.2001517 & 0.0003022 & 1.00000720 & 0.000720 & -0.00118767 & -0.0006598 \\
2 & 100.4003034 & 0.0003022 & 0.02000011 & 0.000550 & 9.98068456 & -0.1931544 \\
3 & 150.6004551 & 0.0003022 & 0.09999896 & -0.001040 & 19.99566769 & -0.0216616 \\
4 & 200.8006068 & 0.0003022 & 0.00999794 & -0.020600 & 29.97587054 & -0.0804315 \\
5 & 251.0007585 & 0.0003022 & 0.04999709 & -0.005820 & 39.99421845 & -0.0144539 \\
6 & 301.2009102 & 0.0003022 & 0.00499651 & -0.069800 & 49.97821918 & -0.0435616 \\
7 & 351.4010619 & 0.0003022 & 0.03999644 & -0.008900 & 59.99594266 & -0.0067623 \\
9 & 451.8013653 & 0.0003022 & 0.02999711 & -0.009633 & 80.00045400 & 0.0005675 \\
11 & 552.2016687 & 0.0003022 & 0.01999984 & -0.000800 & 100.01210024 & 0.0121002 \\
\hline
\end{tabular}

Table. 4 The result of adaptive artificial neural network algorithm

\begin{tabular}{ccccccc}
\hline $\begin{array}{c}\text { Harmonic } \\
\text { number }\end{array}$ & \multicolumn{2}{c}{ Harmonic frequency } & \multicolumn{2}{c}{ Harmonic amplitude } & \multicolumn{2}{c}{ Harmonic phase } \\
Frequency & $\begin{array}{c}\text { Relative } \\
\text { error }\end{array}$ & Amplitude & $\begin{array}{c}\text { Relative } \\
\text { error }\end{array}$ & Phase & $\begin{array}{c}\text { Relative } \\
\text { error }\end{array}$ \\
\hline 1 & 50 & -0.3984 & 0.99854 & -0.146 & 0.08584 & 0.2384 \\
2 & 100 & -0.3984 & 0.01675 & -16.250 & 23.0349 & 130.3490 \\
3 & 150 & -0.3984 & 0.09833 & -1.670 & 24.0217 & 20.1085 \\
4 & 200 & -0.3984 & 0.00961 & -3.900 & 52.8324 & 76.1080 \\
5 & 250 & -0.3984 & 0.04982 & -0.360 & 46.9546 & 17.3865 \\
6 & 300 & -0.3984 & 0.00595 & 19.000 & 82.3320 & 64.6640 \\
7 & 350 & -0.3984 & 0.04043 & 1.075 & 68.2527 & 13.7545 \\
9 & 450 & -0.3984 & 0.03061 & 2.033 & 89.2676 & 11.5845 \\
11 & 550 & -0.3984 & 0.02023 & 1.150 & 100.9942 & 0.9942 \\
\hline
\end{tabular}

As can be seen from the simulation results, the proposed method of measuring harmonic frequency fluctuation has better adaptability, by the white noise signal interference, the precision for each harmonic amplitude and phase angle is high.

\section{Conclusions}

This paper presents a novel triangular basis function neural network algorithm and its convergence theorem. Combined with windowed function interpolation algorithm, we make a harmonic analysis of power system. The above simulation simulation calculations indicates that, in the non-synchronous sampling and the case of non-integral period truncation and white noise, etc., the proposed method is of high accuracy for harmonic measurement. The neural network algorithm requires small amount of data (only one fundamental cycle sample data), and is fast convergence (the above example only 6th iteration can converge). Therefore, the algorithm has a higher value in the power system harmonic measurement. 


\section{References}

[1] Zhang Yibin, Wang Xiaohua, He Yigang. Study on harmonic analysis in power systems based on neural network. Journal of Hunan University (Natural Sciences), Volume 32, Issue 6 (2005), P. 61-64.

[2] Zhao Wenchun, Ma Weiming, Hu An. FFT algorithm with high accuracy for harmonic analysis in the electric machine. Proceedings of t he CSEE, Volume 21, Issue 12 (2001), P, 83-87.

[3] Wei Renyong, Li Zhiyong. Measurement of harmonics in power system based on artificial neural network. Power System Technology, Volume 23, Issue 12 (1999), P. 20-23. 